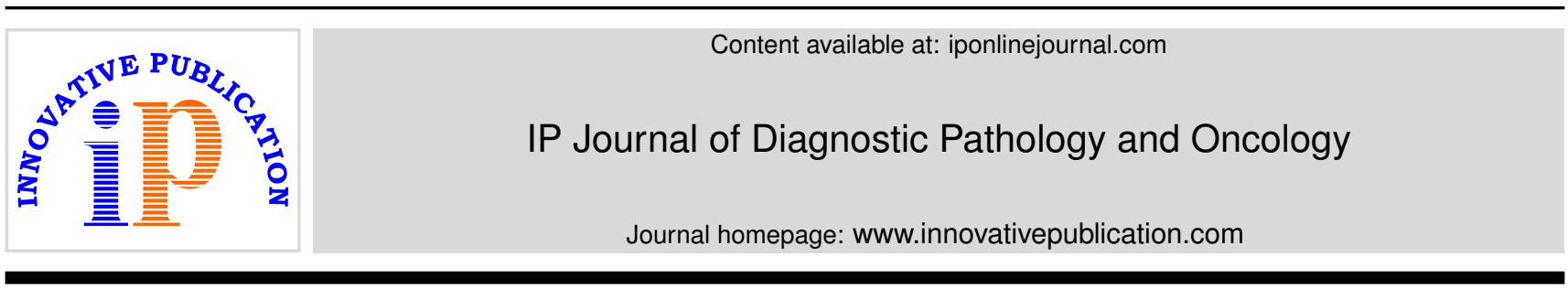

Original Research Article

\title{
Assessment of Dysphagia and pain intensity among patients undergoing chemoradiotherapy: A comparative study among laser and control group
}

\author{
Shantaling Nigudgi ${ }^{1}$, Donald Fernandes ${ }^{2, *}$ \\ ${ }^{1}$ Dept. of Radiation Oncologist, HCG Cancer Center Kalaburagi, Gulbarga, Karnataka, India \\ ${ }^{2}$ Shirdi Sai Cancer Hospital, Kasturba Medical college, Manipal, Karnataka, India
}

\section{A R T I C L E I N F O}

Article history:

Received 18-01-2020

Accepted 30-01-2020

Available online 29-02-2020

\section{Keywords:}

Dysphagia

Pain Intensity

Visual Analogue Scale

Functional Impairment Scale

Laser therapy

\begin{abstract}
A B S T R A C T
Introduction and objective: The present study was undertaken to assess the Dysphagia and pain intensity among patients undergoing chemoradiotherapy.

Materials and Methods: This was a prospective comparative study conducted from March 2009 to August 2010. A total of 109 patients with oral and oropharyngeal cancer were included in the study. The placebo group received sham treatment wh ereas Laser group patients were treated with Low Level Helium-Neon Laser at 6 anatomical sites in the oral cavity prior to Radiotherapy for 45 days. Severity of Dysphagia was assessed daily using Functional Impairment Scale (FIS). Patients were asked for the type of food they were able to take and related difficulty in swallowing that food. Severity of Oral Pain was assessed each day of treatment using The Visual Analogue Scale (VAS) 0 to 10 and Verbal scale were used to describe their pain in terms of various levels and these values were coded for statistical analysis. SPSS 18.0 version was used for statistical analysis.

Results: All the patients were above 40 years of age in laser and placebo group respectively. Nearly $3 / 4$ th of the patients developed a mild degree of dysphagia by the end of first week. At the completion of treatment, $44 \%$ of the patients in the study arm had grade 4 dysphagia, compared to $60 \%$ in the placebo arm. As per the pain, there was a steady increase in the VAS scores from 2 nd week onwards. At the completion of treatment, $50 \%$ of the patients in the control arm had pain scores more than or equal to 7 , while only $16 \%$ in the study arm had severe pain. Both the tools revealed statistically significant results, with the patients in the laser arm experiencing much lower pain during the course of treatment when compared to the placebo arm.

Conclusion: The Laser group experienced less pain and dysphagia while on treatment, compared to the placebo arm. The difference was statistically significant.
\end{abstract}

() 2020 Published by Innovative Publication. This is an open access article under the CC BY-NC-ND license (https://creativecommons.org/licenses/by/4.0/)

\section{Introduction}

Head and neck cancer are general term applied for a group of malignancies with similar natural history, arising from the epithelial lining of the upper aero-digestive tract. Head and neck carcinoma are among the commonest cancers diagnosed in India. ${ }^{1}$ Head and Neck cancers are broadly classified based on their anatomic site of origin into cancers of oral cavity, oropharynx, hypopharynx, and larynx. Worldwide, an estimated 644,000 new cases of

\footnotetext{
* Corresponding author.

E-mail address: intellects19@yahoo.com (D. Fernandes).
}

head and neck cancers are diagnosed each year, with twothirds of these cases occurring in developing countries. However, Oro-pharyngeal cancers account for a small percentage of newly diagnosed cancers in the developed countries. The estimated number of new head and neck cancer cases (excluding skin cancer) in the United States in 2006 represented only $2.9 \%$ of the total new cancer cases. $^{2}$ In contrast, these cancers constitute a major health problem in India accounting for $23 \%$ of all cancers in males and $6 \%$ in females. For many primary sites of head and neck cancer, cigarette and other tobacco use is associated with a significantly increased risk. Alcohol has also been 
implicated as a causative factor, and the effects of alcohol and tobacco seem to be synergistic. ${ }^{3}$

Surgery and Radiotherapy are the only curative treatment options in the management of head and neck squamous cell carcinomas. Chemotherapy by itself usually fails to achieve good response rates, and cannot be considered as a definitive treatment. While single modalities of treatment can achieve excellent cure rates in early stage disease, the results in the advanced stages remain poor. Because of this, combined modality treatment strategies are often employed in higher stage disease. Factors such as age, sex, tumor site, tumor -lymph node-metastasis (TNM) stage and histologic grade may help to guide treatment decisions. Better treatment outcomes in recent times have come at the expense of increased patient morbidity, notably an increase in severe mucositis, dysphagia and pain intensity. Radiation toxicity is classified as acute, sub-acute and late, based on the time period in the development of the particular toxicity. Acute toxicity generally manifests during or within 3 months of the completion of treatment. On the other hand, chronic toxicity is a term that refers to side effects developing more than 9 months after the treatment completion. ${ }^{4}$ The scope of this study is limited to the study of acute toxicity, principally dysphasia and pain intensity that develops during the course of treatment. With this background the present study was undertaken to Assess the dysphagia and pain intensity among patients undergoing chemoradiotherapy.

\section{Materials and Methods}

This was a prospective comparative study conducted from March 2009 to August 2010. 163 head and neck cancer patients who attended the clinic were screened as per the criteria. Among them 109 were included in the study after obtaining a written informed consent. The patients were randomly allocated to laser (54) and placebo (55) group using computer generated random program and matched for age and site of the primary tumour. The study was completed by all the patients. Three tier blinding was carried out at the patients, assessor and data analysing statistician.

The inclusion criteria of the patients were age 18 years and above with Eastern Cooperative Oncology Group (ECOG) performance score of 2 or less, scheduled to undergo curative concurrent chemoradiotherapy for primary oral and oropharyngeal cancers. Whereas Patients with Trismus, ECOG performance score of more than 2, medically unfit, presence of distant metastasis, medically compromised conditions, prior radiation or neo-adjuvant chemotherapy and patients not receiving high dose ( $>50 \mathrm{~Gy})$ radiation to the oral cavity were excluded from the study. Ethical clearance was obtained from the hospital ethical committee before commencing the trial and written informed consent was obtained from all the participants.
The placebo group received sham treatment (i.e. simple red light) while Laser group patients received LLLT. Both groups received the similar oral care and hygiene protocol which included frequent mouth wash with sodium bicarbonate, bland soft diet. Dental consultation and oral treatment prior to radiation if necessary. A single operator who was experienced in the delivery of LLLT field treated all the patients. Patients in Laser group were treated with Low Level Helium-Neon Laser at 6 anatomical sites in the oral cavity prior to Radiotherapy for 45 days. During the laser or the placebo treatment, the patient and the therapist wore wavelength specific protective eye goggles.

An experienced radiation oncologist who was unaware for the trial intervention group did the clinical assessment of all the patients. The evaluation was done during the course of the treatment, on a daily basis, and the highest recorded value in that week was taken as the weekly measure. Severity of Dysphagia was assessed daily using Functional Impairment Scale (FIS). The FIS is graded as $1=$ able to eat solid foods, $2=$ able to eat soft foods, $3=$ able to drink liquids, $4=$ oral alimentation not possible. Patients were asked for the type of food they were able to take and related difficulty in swallowing that food. Severity of Oral Pain was assessed each day of treatment using The Visual Analogue Scale (VAS) Patients were asked to illicit the maximum pain experienced by them by considering $0=$ Absence of pain, $10=$ Maximum pain imagined by them. Verbal scale was used for Patients to describe their pain in terms of various levels used in this scale and these values were later coded for statistical analysis. Worst possible $=4$, severe $=3$, moderate $=2$, low $=1$ and none $=0$. The above 2 scales were used to ensure greater comparability in the subjective assessment of pain.

\subsection{Statistical analysis}

SPSS version 18 was used for statistical analysis. Descriptive statistics was calculated for numeric over a period of one week, 7 values (treatment duration being 7 weeks) were thus obtained for laser and control group individually. These were compared using test of repeated measures. Chi-square test was also used to determine if there is any association between categorical variables from two or more groups. Verbal scale was assessed similar to numeric rating scale using test of repeated measures. Maximum functional impairment per week was calculated which were then compared using test of repeated measures. $\mathrm{P}$ value less than or equal to 0.05 was considered significant.

\section{Results}

This was a comparative longitudinal study carried between March 2009 to August 2010. A total of 109 patient were included in the study they were divided into laser and placebo group under the study. The mean age of 
the patients in the study was $56.90 \pm 11.97$ years and $55.18 \pm 10.94$ years in laser and placebo group respectively. Male patients (80\%) were comparatives more than females. Sex distribution, oral mali gnancy and Oro- pharyngeal malignancy between the groups were similar.

As per the staging of the disease Patient with Stage I disease were not included whereas Stage IV disease was more seen among placebo group. As per the Functional Impairment scale over the course of treatment in the two arms, nearly 3/4th of the patients had developed a mild degree of dysphagia as early as the end of first week. The progress in dysphagia was similar in between the arms, and by 3rd week nearly $50 \%$ of patients had moderate to severe dysphagia. Also, the severity of dysphagia continued to progress with treatment. At the completion of treatment, $44 \%$ of the patients in the study arm had grade 4 dysphagia, compared to $60 \%$ in the placebo arm. Despite this moderately large difference, they were not statistical significance $(\mathrm{p}=0.45)$. There was a near-parallel progression in the Functional Impairment scores, with a small difference in between the two arms, starting from the 2nd week, favo uring the Laser arm. There was no statistical difference in the functional impairment scores between the two arms $(\mathrm{p}=0.41)$. (Table 1$)$

The pain experienced by the patients during the treatment was analysed using both the Visual analogue scale (VAS) and the verbal pain scores. There was a steady increase in the VAS scores from 2nd week onwards in the placebo group. On the other hand, most patients in the laser group experienced a more gradual increase in the pain scores. A lesser incidence of severe pain was also noted in the laser arm from 3rd week onwards. (Table 2). As per the incidence of severe pain 8-11 patients in the study arm experienced severe pain beyond the 4th week of treatment, while in the control arm increasing numbers of patients had severe pain as they neared the completion of treatment. At the completion of treatment, $50 \%$ of the patients in the control arm had pain scores more than or equal 7, while only $16 \%$ in the study arm had severe pain. Both the tools revealed similar results, with the patients in the laser arm experiencing much lower pain during the course of treatment when compared to the placebo arm. The difference was statistically significant $(\mathrm{p}<0.001)$.

Both the arms experienced loss of weight over the course of treatment. The mean weight of the patients at the start of treatment was $54.27 \mathrm{~kg}$ in the study arm and $52.6 \mathrm{~kg}$ in the control arm. At the end of treatment, the mean weight was 49.62 and $48.49 \mathrm{kgs}$, respectively. The weight loss was similar between the two arms, and there was no statistical significance in the results $(\mathrm{p}=0.78)$. The patients in the two arms were compared based on the stage of their disease on all the parameters. All the parameters, namely Functional impairment scales, Pain evaluation by Visual analogue scale and verbal scale, and weight loss, were similar in patients in both groups with stage II disease, and no statistical significance was noted. As seen in subgroups with stage II disease, there was no difference in the outcomes in the laser and the placebo arms in stage III. The outcomes were significantly different between the two arms among Patients with stage IV disease, favou ring the laser arm. While the Functional Impairment scales and Weight loss were similar in both groups, the pain scores were significantly less in the laser group $(\mathrm{p}<0.001)$ (Table 3$)$

\section{Discussion}

Both the study and the placebo arms were comparable in most of the demographic aspects. The great proportion of males in oral cancer could be a direct consequence of sex distribution of tobacco habits. In this study the severity of pain and swallowing difficulties were compared between the laser and control groups. The principle behind using lasers is that it is known to accelerate wound healing and has antiinflammatory action. Wound healing is one of the most studied aspects of low energy lasers. In studies of fibroblast responses to laser, increased cell division or increased collagen production have been reported in gingival tissues, He-Ne laser applications have stimulated DNA synthesis of myofibroblasts without any degenerative changes, and have transformed fibroblasts into myofiboblasts, which may promote wound healing. ${ }^{5}$ With regard to pain relief, one proposed mechanism is modulation of nociception by modification of nerve conduction via the release of endorphins and enkephalins.

In a phase III randomized double-blind placebocontrolled clinical trial carried to determine the efficacy of low level laser therapy for the prevention of morbidities in patients undergoing hematopoietic cell transplantation, Mucositis and oral pain was measured on days $0,4,7,11$, 14,18 , and 21 post HCT. The $650 \mathrm{~nm}$ wavelength reduced the severity of oral mucositis and pain scores. Low level laser therapy was well-tolerated and no adverse events were noted. Laser therapy also reduced the time of oral pain from 5.64 to 2.45 days $(\mathrm{P}=0.04)$, and decreased the consumption of morphine $(\mathrm{P}=0.07) .{ }^{6,7}$ Which are similar to our finding were laser group showed a significant lower pain intensity as compared to placebo group.

In a Randomized, Placebo-controlled Study 23 patients receiving only Radiotherapy for head and neck cancer were randomized into LLLT(11) and Placebo(12) groups, The pain score after each laser or placebo application was significantly lower $(p<0.006)$ in the LLLT group during the same period. ${ }^{7}$ A Randomized, controlled Study 24 patients receiving only Radiotherapy for oral cancer were randomized into LLLT(11) and Control(13) groups, the patients were evaluated on each day of treatment for pain severity (NRS), functional impairment (FIS), and oral mucositis (RTOG) and were followed until the end of cancer treatment. Results of this study concluded that use of LLLT 
Table 1: Progression of Dysphagia among the two groups

\begin{tabular}{|c|c|c|c|c|c|c|c|c|}
\hline \multirow[b]{2}{*}{ Dysphagia (FIS) } & \multicolumn{4}{|c|}{ Laser $(\%)$} & \multicolumn{4}{|c|}{ Placebo (\%) } \\
\hline & 1 & 2 & 3 & 4 & 1 & 2 & 3 & 4 \\
\hline Week1 & 78 & 18 & 0 & 4 & 70 & 19 & 11 & 0.00 \\
\hline Week2 & 60 & 31 & 5 & 4 & 38 & 34 & 23 & 5 \\
\hline Week3 & 18 & 36 & 28 & 18 & 7 & 31 & 45 & 17 \\
\hline Week4 & 7 & 26 & 35 & 32 & 2 & 15 & 53 & 30 \\
\hline Week5 & 8 & 22 & 30 & 40 & 2 & 11 & 40 & 47 \\
\hline Week6 & 7 & 18 & 31 & 44 & 2 & 9 & 34 & 55 \\
\hline Week7 & 9 & 16 & 31 & 44 & 2 & 6 & 32 & 60 \\
\hline
\end{tabular}

Table 2: Comparison of pain score using VAS assessment

\begin{tabular}{|c|c|c|c|c|}
\hline \multicolumn{3}{|c|}{ VAS Scores-median values } & \multicolumn{2}{|c|}{ VAS Scores- severe pain (scores $\geq 7$ ) } \\
\hline Week & Laser (50) & Placebo (50) & Laser (50) & Placebo (50) \\
\hline 1 & $0(0-5)$ & $0(0-6)$ & 0 & 0 \\
\hline 2 & $0(0-5)$ & $1(0-7)$ & 0 & 1 \\
\hline 3 & $0(0-8)$ & $3(1-9)$ & 5 & 9 \\
\hline 4 & $2(0-8)$ & $5(1-8)$ & 8 & 16 \\
\hline 5 & $3(0-8)$ & $5(1-9)$ & 11 & 14 \\
\hline 6 & $3(0-8)$ & $7(1-9)$ & 8 & 18 \\
\hline 7 & $3(0-8)$ & $7(0-9)$ & 7 & 25 \\
\hline
\end{tabular}

Table 3: Stage of disease and outcome in terms of Functional impairment and Pain Intensity

\begin{tabular}{lll}
\hline Stage of disease & p value measured & \\
II & Functional Impairment & Pain (by VAS) \\
III & 0.18 & 0.001 \\
IV & 0.25 & 0.03 \\
& 0.19 & 0.01 \\
\hline
\end{tabular}

reduce the severity of oral mucositis, severity of pain, and functional impairment ${ }^{8}$ which is comparable to our findings

Curative laser application seems less successful than prophylactic laser application though the reason is not entirely clear. There were no adverse effects noted with the use of low level laser, though it is important to realize the importance of preventing retinal damage by the use of wavelength specific goggles. This is consistent with the previous reports. ${ }^{8,9}$ The incidence of severe pain in the laser group patients was less compared to Placebo group. The ability to swallow was better maintained with LLLT, which is similar to those reported by Bensadoun et al. and similar study ${ }^{10,11}$ However, study by Cowen et al. ${ }^{12}$ equal numbers in the laser and control groups required parenteral nutrition. The authors did not mention the reason behind this. None of our patients required paraenteral feeds. Twenty three patients required Ryle's tube feeds, slightly more in the control group (10 vs. 13). This correlates well with the dysphagia experienced between the two arms. Therefore, our study shows greater advantage of lasers.

Pain was measured on two scales, Visual Analogue scale (VAS) and verbal scale (VS). VAS is more accurate as compared to VS as it takes into consideration minute variations of pain. It was observed that pain severity was mild for the laser group and moderate for placebo group. $(p=0.001)$. The results of the pain assessment by Visual Analogue scale and Verbal scale in our study were comparable, and the pain scores were consistently lower in the laser group by a value of 1.5 beyond the 3rd week of treatment. Bensadoun et al. ${ }^{13}$ also reported the similar results in their study. A study done in 1999 also reported substantial differences in mean pain rank and mean pain score $(\mathrm{P}<0.001){ }^{13}$ Our results support the contention that laser therapy applied prophylatically during radiotherapy can reduce the severity of mucositis, severity of pain and functional impairment.

Possible mechanism of the healing effects seems to be related to an activation of energy production in the mitochondria. ${ }^{14}$ It has also been demonstrated that LLLT results in a rapid generation of myofibroblasts from fibroblasts and the fibroblast growth factors seems to play a role in epithelial repair and cryoprotection. ${ }^{15}$ Also it has been suggested that, LLLT may be capable of detoxification $\mathrm{O} 2$ free radicals during cytoreductive therapy. ${ }^{16}$ Some studies have attributed the enhancement of wound healing and pain relief potential of LLLT to microscopic findings as increased cell division and modification of nerve conduction via the release of 
endorphins and enkephalins. ${ }^{12,13}$ However, as noted above, the placebo arms had more patients with stage IV disease. As this could confound the final result, sub group analyses were done.

\section{Conclusion}

The patients receiving laser had lesser incidence of dysphagia compared to the placebo arm. However, the statistical significance was not reached. A larger study needs to be done to confirm this clinically important end point. The results of the pain assessment by Visual Analogue scale (VAS) and Verbal scale (VS) were comparable, and the pain scores were consistently lower in the laser group, Weight loss was similarly experienced in both the arms.

\section{Source of funding}

None.

\section{Conflict of interest}

None.

\section{References}

1. Jemal A, Bray F, Center MM, Ferlay J, Ward E, et al. Global cancer statistics. CA Cancer J Clin. 2011;61:69-90.

2. Murphy SL, Kochanek KD, Xu J, Heron M. Deaths: Final Data for 2012. National Vital Statistics Rep. 2015;63(9).

3. Gilpin NW, Koob GF. Neurobiology of alcohol dependence: Focus on motivational mechanisms. Alcohol Res Health. 2008;31:185-195.

4. Epstein JB, Emerton S, Kolbinson DA. Quality of life and oral function following radiotherapy for head and neck cancer. Head Neck. 1999;21:1-11.

5. Pourreau-Schneider N, Ahmed A, Soudry M. Helium-neon laser treatment transforms fibroblasts into myofibrolasts. Am J Pathol. 1990;137:171-178.

6. Mark M, Schubert FP. A phase III randomized double-blind placebo-controlled clinical trial to determine the efficacy of low level laser therapy for the prevention of oral mucositis in patients undergoing hematopoietic cell transplantation. Support Care Cancer. 2007; 15:1145-1154.

7. Jaguar GC, Prado JD, Nishimoto IN. Low-energy laser therapy for prevention of oral mucositis in hematopoietic stem cell transplantation. Oral Dis. 2007;13:538-543.

8. Kuhn A, Porto F, Miraglia P, Brunetto AL. Low-level infrared laser therapy in chemotherapy-induced oral mucositis. J Pediatr Hematol Oncol. 2009;31:33-37.

9. Arora H, Maiya AG, Keerthilatha MP, Sagar MS, Fernandes DJ. Efficacy of He-Ne Laser in the prevention and treatment of radiotherapy-induced oral mucositis in oral cancer patients. Oral Surg Oral Med Oral Pathol Oral Radiol Endod. 2008;105:180-186.

10. Bensadoun RJ, Ciais G, Darcourt V, Franquin JC, Cheynet C, et al. Low energy laser in the prevention of radiation induced mucositis: a phase II randomized multicentre study for patients with head and neck cancer. Supportive Care Cancer. 1999;7:SO-13.

11. Migliorati C, Massumoto C, De P, Eduardo F. Low-energy laser therapy in oral mucositis. J Oral Laser Appl. 2001;1:97-101.

12. Cowen D, Tardieu C, Schubert M. Low energy He-Ne laser in the prevention of oral mucositis in patients undergoing bone marrow transplant: results of double blind randomized trial. Int J Radiat Oncol Biol Phys. 1997;38:697-703.

13. Bensadoun RJ, Franquin JC, Ciais G. Low-energy He-Ne laser in the prevention of radiation-induced mucositis. Support Cancer Care. 1999;7:244-252.

14. Bortoletto R, Silva NS, Zangaro RA. Mitochondrial membrane potential after low-power laser irradiation. Lasers Med Sci. 2004;18:204-206.

15. Pourreau-Schneider N, Ahmed A, Soudry M. Helium-neon laser treatment transforms fibroblasts into myofibrolasts. Am J Pathol. 1990;137:171-178.

16. Gate L, Paul J, Ba GN. Oxidative stress induced in pathologies: the role of antioxidants. Biomed Pharmacother. 1999;53:169-180.

\section{Author biography}

Shantaling Nigudgi Consultant

Donald Fernandes Professor and Unit Chief

Cite this article: Nigudgi S, Fernandes D. Assessment of Dysphagia and pain intensity among patients undergoing chemoradiotherapy: A comparative study among laser and control group. IP J Diagn Pathol Oncol 2020;5(1):13-17. 